\title{
Towards Inclusion: a Guidebook for Championing Diversity for Internal Medicine Chief Medical Residents
}

\author{
Sneha Thatipelli, $M D^{\top} \odot$, Nkiru Osude, $M D^{2}$, and Quentin R. Youmans, $M D^{3}$ \\ 'Department of Medicine, Northwestern University Feinberg School of Medicine, Chicago, IL, USA; ${ }^{2}$ Department of Medicine, Loyola University \\ Medical Center, Maywood, IL, USA; ${ }^{3}$ Division of Cardiology, Department of Medicine, Northwestern University Feinberg School of Medicine, \\ Chicago, IL, USA
}

J Gen Intern Med 36(6):1761-4

DOI: $10.1007 / \mathrm{s} 11606-021-06782-\mathrm{w}$

(c) Society of General Internal Medicine 2021

$\mathrm{R}$ esidency programs recognize the lack of physicians of color and have made efforts to increase diversity within the ranks of graduate medical education. ${ }^{1,2}$ While prioritizing diversity is important, it cannot be attained and maintained without an equal emphasis on inclusion. ${ }^{3}$ Inclusive practices create a sense of belonging and worth for all trainees ${ }^{4-6}$, and can lead to improved individual and overall organizational performance. ${ }^{7,8}$ As current and former chief medical residents (CMRs), we argue that the responsibility to create systems that value these practices must fall upon residency leadership. For internal medicine (IM) residency programs, the CMR could serve as an untapped champion. Historically, CMRs are recent graduates of the programs or current senior residents and are privy to the structures of the residency program. They function as junior faculty members focusing on education, administrative tasks, mentorship, and leadership. ${ }^{9}$ This near-peer leadership role allows CMRs to serve as liaisons between program leadership and residents while championing new improvement initiatives.

With this perspective, we aim to equip residency programs to harness the role of CMR (both underrepresented in medicine and not) to create an inclusive environment, design inclusive education, and institute recruitment practices that champion diversity in IM residency programs. An inclusive environment is one that supports trainees. It should foster mutual respect for one another and focus on integration of individual perspectives and experiences. ${ }^{3}$ An inclusive education is one that is ingrained into the fabric of residency, imbuing learners with a holistic perspective on the impacts of clinical care and unearthing implicit biases during all aspects of structured and unstructured training. ${ }^{5}$ This includes

Dr. Thatipelli and Dr. Osude contributed equally as co-first authors.

Received October 18, 2020

Accepted March 30, 2021

Published online April 12, 2021 anti-racist education, defined as didactics that take an active stance to combat racism in all forms. ${ }^{10}$

\section{INCLUSIVE ENVIRONMENT}

Fostering inclusion in residency begins by creating an inclusive environment. ${ }^{6}$ Unfortunately, residents who are underrepresented in medicine (UIM) are victims of institutional racism. ${ }^{11}$ They experience macroaggressions, microaggressions, and bias that can affect their performance. ${ }^{11}$ These can occur in everyday activities including the evaluation of patients, conversations with peers, discussions on rounds, or feedback with attending physicians. ${ }^{11}$ Racially motivated adverse behaviors often go unreported due to the hierarchy of medicine and fears of retribution, vulnerability, and inaction. ${ }^{11-13}$ These experiences run counter to inclusion and make it hard for UIM residents to thrive. As CMRs, our near-peer status puts us in a unique position to prevent and/ or interrupt these experiences. Therefore, we must take the steps to analyze the current environment, create safe spaces, and advocate for inclusion.

\section{Understand the Current Environment}

Different trainees may experience the same residency culture differently. To fully understand the current environment, programs must take into account the individual impact that the "hidden curriculum," burnout, implicit bias, and explicit bias have on its trainees. Learning environments that unintentionally expose trainees to unprofessional and unethical behavior have been shown to further burnout and cynicism and be detrimental to professional development. ${ }^{14,}{ }^{15}$ In addition, symptoms of burnout are highest among Black residents who experience high levels of implicit and explicit bias. ${ }^{16}$ CMRs should work with leadership to elicit a diverse array of housestaff perceptions of the learning environment and inclusion via town halls, UIM focus groups, and/or inclusion surveys to obtain a full understanding of the perceptions of the current environment. ${ }^{17,18}$ Programs need to create an avenue for continuous anonymous feedback ${ }^{19,20}$ regarding the environment throughout the year (e.g., suggestion boxes and online surveys) to encourage residents to share their honest perceptions without fear of retribution. By discussing aspects 
of the program that have been identified as exclusive in regular open fora ${ }^{20}$, programs can create a shared understanding of barriers to an inclusive environment. This can then be leveraged such that every resident has a hand in creating an improved and more inclusive residency culture.

\section{Target High-Risk Areas for Intervention}

The spaces we inhabit while at work can be our sanctuaries or our silos. Although attempts should be made to improve all areas within the program, we have found success in targeting interventions to promote inclusion in high-risk areas such as team rooms where trainees spend a majority of their time. CMRs should engage in frequent informal check-ins in these high-risk areas to understand concerns that may not be elicited in large group settings (i.e., via "candy rounds"). Inclusion can be reinforced visually through signage in shared and virtual spaces (e.g., "Hate Has No Home Here," lapel pins celebrating LGBTQ+ and pronouns on white coats, BLM, and White Coats for Black Lives).

\section{Create a Safe Space for UIM Residents}

Program leadership should identify faculty members (UIM and non-UIM) who can serve as mentors for UIM residents to foster allyship. ${ }^{21} \mathrm{CMRs}$ can encourage relationships among UIM residents by hosting events in which those of underrepresented backgrounds can bond through shared experiences. Programs should collaborate with nearby residency programs as well to achieve the above and expand the creation of safe spaces for UIM residents.

\section{Model Inclusive Behaviors}

Trainees look up to CMRs as role model clinicians and educators. As such, we should model inclusive behaviors and be conscientious. For example, as attendings on service, we should be mindful of our documentation, working to avoid race in one-liners and stigmatizing language, which have been shown to have a negative impact on physician attitudes and outcomes. ${ }^{22}$ We should start self-introductions with pronouns and request the same of trainees and patients. We must also be aware of the implicit bias that exists in trainee evaluations ${ }^{23-}$ ${ }^{26}$, and take special care to avoid them. CMRs should push the department to assess the diversity of invited conference speakers and the breadth of content delivered, conducting regular internal reviews on the demographics of the faculty invited in order to strive for an equitable representation based on gender, race, ethnicity, and faculty position.

\section{INCLUSIVE AND ANTI-RACIST EDUCATION}

An inclusive environment can promote inclusive education, both in content and delivery. Inclusive content revolves around but is not limited to the social determinants of health $(\mathrm{SDOH})$, cultural humility, ability and disability education,
LGBTQ+ health and access, and bias in medicine. It also includes anti-racist education to equip trainees with an understanding of racially motivated disparities. CMRs play a key role in developing the educational curriculum for residents and must leverage this position to incorporate inclusive and antiracist education.

\section{Incorporate Inclusion and Anti-Racism into Regularly Scheduled Didactics}

CMRs should incorporate SDOH and the impact of racism into clinical vignettes during morning reports and noon conferences to demonstrate the effect on physician attitudes and decision-making. We should teach the flaws of race-based medicine (e.g., estimated glomerular filtration rate ${ }^{27}$ ) and discuss disparities in patient outcomes at our own cities and states. $^{28}$ These discussions should lay the foundation for how trainees and thus the future medical community can mitigate gaps in healthcare and work towards actionable strategies to achieve health equity. Whereas current medical education is generally focused on the patient presentation in cisgender and White individuals ${ }^{29}$, we should expand beyond this norm and include patient presentations in diverse populations. ${ }^{30}$ Group didactics (i.e., conference series, journal clubs, book clubs) should be used to teach the history of systemic racism and how it has created systems of oppression and exploitation that have resulted in health disparities.

\section{Equip Residents to Pursue a Career Path of Inclusion}

CMRs can facilitate connections between residents and faculty members with shared interests. If trainees express an interest in a career of diversity and inclusion, CMRs can go a step further beyond what is in the curriculum by exposing interested residents, UIM or not, to mentors immersed in various inclusive efforts apart from academia, such as community engagement, political advocacy, and professional medical societies.

\section{RECRUITMENT OF DIVERSE RESIDENTS}

Academic medicine has historically lacked diversity. ${ }^{31} \mathrm{Al}-$ though there are efforts to improve diversity within residency, the CMR has had an underutilized role in promoting these efforts. We believe the steps discussed above will entice medical students, particularly UIM, to choose IM as a specialty. Still, efforts for diversity recruitment are necessary to ensure that candidates are fairly evaluated based on their diverse skill sets, backgrounds, and experiences.

\section{Incorporate Holistic Review in Recruitment}

Departments should train interviewers and selection committees on the nuts and bolts of holistic review. ${ }^{32}$ We need to amend the application review process such that the breadth of qualities of advocacy, interpersonal skills, and leadership are 
valued and celebrated. ${ }^{32}$ CMRs should be afforded the opportunity to participate as interviewers and reviewers on the selection committee, to serve as proponents of diversity, and to advocate for the value of these humanistic attributes.

\section{Mitigate Bias Through Training and Awareness for All Selection Committee Members}

All CMRs and selection committee members should participate in implicit bias training in order to be aware of and minimize bias in the review process. ${ }^{33}$ Faculty and CMRs need to recognize that explicit bias is both still prevalent and pervasive and call it out when it is observed throughout the recruitment process.

\section{Prioritize Diversity During the Interview Season}

Media (PowerPoints, videos, social media) created by the program should highlight and celebrate diversity and inclusion in all its forms. Effort should be taken to create fora for UIM applicants to meet and interact with UIM residents so they can assess fit and freely inquire about institutional culture. While it is important to make these opportunities available, it is equally important to ensure that all trainees have the freedom to pursue their individual interests and do not feel obligated to do work in the space of diversity, equity, and inclusion as this can contribute to the minority tax. ${ }^{34}$

\section{CONCLUSIONS}

Lastly, being thoughtful about how we preserve this culture of inclusion is key for sustainability. The impact of individual CMRs is limited by the annual turnover of our position unless we leverage our roles as liaisons to collaborate across different levels of leadership for sustainable solutions.

\section{Collaborate for Sustainability}

Departments should use or create formalized positions like Vice Chair and Associate Program Director to become champions for these issues. By setting intentional mission statements to reflect institutional commitment, they can further foster allyship from hospital-wide leadership. ${ }^{5}$ In addition, work towards inclusion should not be completed in silos. Residency leadership should engage with nearby programs that share a similar commitment to inclusion in order to exchange solutions, curricula, and interventions to overcome barriers.

The time is now for us to pause and reimagine the power and responsibility of CMRs. We must choose to utilize this influence to create training environments and curricula which elevate all trainees. We hope that by leveraging our unique position and influence, we will create educational systems that yield future generations of internal medicine trainees who are equipped to be inclusive physicians.
Acknowledgements: The authors would like to thank their residency programs and their residents.

Corresponding Author: Sneha Thatipelli, MD; Department of Medicine, Northwestern University Feinberg School of Medicine, Chicago, IL, USA (e-mail: Sthatipelli09@gmail.com).

\section{Declarations:}

Conflict of Interest: The authors declare that they do not have a conflict of interest.

\section{REFERENCES}

1. Gonzaga AMR, Appiah-Pippim J, Onumah CM, Yialamas MA. A Framework for Inclusive Graduate Medical Education Recruitment Strategies: Meeting the ACGME Standard for a Diverse and Inclusive Workforce. Acad Med. 2020;95(5):710-716.

2. Khan NR, Taylor CM, 2nd, Rialon KL. Resident Perspectives on the Current State of Diversity in Graduate Medical Education. J Grad Med Educ. 2019;11(2):241-243.

3. McDade WA. Increasing Graduate Medical Education Diversity and Inclusion. J Grad Med Educ. 2019;11(6):736-738.

4. Downey SN, van der Werff $\mathbf{L}$, Thomas KM, Plaut VC. The role of diversity practices and inclusion in promoting trust and employee engagement. Journal of Applied Social Psychology. 2015;45(1):35-44.

5. Diaz T, Navarro JR, Chen EH. An Institutional Approach to Fostering Inclusion and Addressing Racial Bias: Implications for Diversity in Academic Medicine. Teach Learn Med. 2020;32(1):110-116.

6. Shore LM, Randel AE, Chung BG, Dean MA, Holcombe Ehrhart K, Singh G. Inclusion and Diversity in Work Groups: A Review and Model for Future Research. Journal of Management. 2011;37(4):1262-1289.

7. Cho S, Mor Barak ME. Understanding of Diversity and Inclusion in a Perceived Homogeneous Culture: A Study of Organizational Commitment and Job Performance Among Korean Employees. Administration in Social Work. 2008;32(4):100-126.

8. Chen $\mathbf{C}$, Tang $\mathbf{N}$. Does perceived inclusion matter in the workplace? Journal of Managerial Psychology. 2018;33(1):43-57.

9. Singh D, MCDonald FS, Beasley BW. Demographic and work-life study of chief residents: a survey of the program directors in internal medicine residency programs in the United States. J Grad Med Educ. 2009;1(1):150-154.

10. Kendi IX. How to be an antiracist. New York: One World; 2019.

11. Osseo-Asare A, Balasuriya L, Huot SJ, et al. Minority Resident Physicians' Views on the Role of Race/Ethnicity in Their Training Experiences in the Workplace. JAMA Netw Open. 2018;1(5):e182723.

12. Nunez-Smith M, Pilgrim N, Wynia M, et al. Health care workplace discrimination and physician turnover. J Natl Med Assoc. 2009;101(12):1274-1282.

13. Liebschutz JM, Darko GO, Finley EP, Cawse JM, Bharel M, Orlander JD. In the minority: black physicians in residency and their experiences. J Natl Med Assoc. 2006;98(9):1441-1448.

14. Dyrbye LN, Thomas MR, Harper W, et al. The learning environment and medical student burnout: a multicentre study. Med Educ. 2009;43(3):274-282.

15. Billings ME, Lazarus ME, Wenrich M, Curtis JR, Engelberg RA. The effect of the hidden curriculum on resident burnout and cynicism. J Grad Med Educ. 2011;3(4):503-510.

16. Dyrbye L, Herrin J, West CP, et al. Association of Racial Bias With Burnout Among Resident Physicians. JAMA Netw Open. 2019;2(7):e197457.

17. Kitzinger J. Qualitative research. Introducing focus groups. BMJ. 1995;311(7000):299-302.

18. Al-Busaidi ZQ. Qualitative research and its uses in health care. Sultan Baboos Univ Med J. 2008;8(1):11-19.

19. Gong Z, Zhang $\mathbf{N}$. Using a Feedback Environment to Improve Creative Performance: A Dynamic Affect Perspective. Front Psychol. 2017;8:1398.

20. Frampton A, Fox F, Hollowood A, et al. Using real-time, anonymous staff feedback to improve staff experience and engagement. BMJ Qual Improv Rep. 2017;6(1).

21. Odom KL, Roberts LM, Johnson RL, Cooper LA. Exploring obstacles to and opportunities for professional success among ethnic minority medical students. Acad Med. 2007;82(2):146-153. 
22. Chapman EN, Kaatz A, Carnes M. Physicians and implicit bias: how doctors may unwittingly perpetuate health care disparities. J Gen Intern Med. 2013;28(11):1504-1510.

23. Rojek AE, Khanna R, Yim JWL, et al. Differences in Narrative Language in Evaluations of Medical Students by Gender and Under-represented Minority Status. J Gen Intern Med. 2019;34(5):684-691.

24. Klein R, Julian KA, Snyder ED, et al. Gender Bias in Resident Assessment in Graduate Medical Education: Review of the Literature. $J$ Gen Intern Med. 2019;34(5):712-719.

25. Klein R, Ufere NN, Rao SR, et al. Association of Gender With Learner Assessment in Graduate Medical Education. JAMA Netw Open. 2020;3(7):e2010888.

26. Axelson RD, Solow CM, Ferguson KJ, Cohen MB. Assessing implicit gender bias in Medical Student Performance Evaluations. Eval Health Prof. 2010;33(3):365-385.

27. Vyas DA, Eisenstein LG, Jones DS. Hidden in Plain Sight Reconsidering the Use of Race Correction in Clinical Algorithms. New England Journal of Medicine. 2020.

28. Eberly LA, Richterman A, Beckett AG, et al. Identification of Racial Inequities in Access to Specialized Inpatient Heart Failure Care at an Academic Medical Center. Circ Heart Fail. 2019;12(11):e006214.
29. Nolen L. How Medical Education Is Missing the Bull's-eye. $N$ Engl J Med. 2020;382(26):2489-2491.

30. Sekoni AO, Gale NK, Manga-Atangana B, Bhadhuri A, Jolly K. The effects of educational curricula and training on LGBT-specific health issues for healthcare students and professionals: a mixed-method systematic review. J Int AIDS Soc. 2017;20(1):21624.

31. Yousif H, Ayogu N, Bell T. The Path Forward - An Antiracist Approach to Academic Medicine. N Engl J Med. 2020.

32. Conrad SS, Addams AN, Young GH. Holistic Review in Medical School Admissions and Selection: A Strategic, Mission-Driven Response to Shifting Societal Needs. Acad Med. 2016;91(11):1472-1474.

33. Capers Qt, Clinchot D, McDougle L, Greenwald AG. Implicit Racial Bias in Medical School Admissions. Acad Med. 2017;92(3):365-369.

34. Rodriguez JE, Campbell KM, Pololi LH. Addressing disparities in academic medicine: what of the minority tax? BMC Med Educ. 2015;15:6.

Publisher's Note: Springer Nature remains neutral with regard to jurisdictional claims in published maps and institutional affiliations. 